\title{
Photoselective Metal Deposition on Amorphous Silicon p-i-n Solar Cells
}

\author{
E. S. Kooij,, M. Hamoumi, ${ }^{a}$ J. J. Kelly,,a and R. E. I. Schropp ${ }^{\natural}$ \\ ${ }^{2}$ Department of Condensed Matter, ${ }^{\circ}$ Department of Interface Physics, Debye Institute, \\ Utrecht University, 3508 TA Utrecht, The Netherlands
}

\section{ABSTRACT}

A novel method is described for the patternwise metallization of amorphous silicon solar cells, based on photocathodic deposition. The electric field of the $p-i-n$ structure is used for the separation of photogenerated charge carriers. The electrons are driven to the interface of the $\mathrm{n}^{+}$-layer with the solution where they reduce metal ions to metal. The large difference between the conductivity of dark and illurninated areas and the high sheet resistance of the n-type layer makes it possible to define a metal pattern by selective illumination. It is shown that both nickel and gold patterns can be deposited using this method. After annealing, an ohmic nickel contact is formed and the cell exhibits good photovoltaic characteristics.

Thin film solid-state photovoltaic cells, such as a-Si:H solar cells, are generally provided with patterned ohmic contacts by evaporating a metal (e.g., $\mathrm{Ag}$ or $\mathrm{Al}$ ) in vacuum which is then selectively removed by laser ablation or photolithographic techniques. An alternative method is selective evaporation of the metal using a shadow mask. Such methods can be costly, especially for mass production of large area solar panels.

In the past we have shown that p-type semiconductors can be selectively metallized by photocathodic deposition from solution.' The semiconductor in contact with the electrolyte forms a Schottky junction, ${ }^{2}$ which has a low reverse current in the dark. For systems with a negative redox potential, the acceptor levels of the metal ion correspond to the conduction band of the semiconductor and, consequently, conduction band electrons are required for the reduction of the metal salt. Electrons and holes, generated by illumination, are separated by the electric field of the depletion layer. At the solution interface the electrons reduce metal ions to produce a metal or alloy on the illuminated areas. High resolution patterns can be produced in this way. ${ }^{1}$

A crystalline silicon p-n junction, the n-type layer of which is in contact with a solution containing an electron acceptor, resembles a p-type semiconductor; the reverse current in the dark is very low, while electrons and holes, generated under illumination, are separated in the internal field of the junction. The hole migrates to the $p$ type material and contributes to the photocurrent, while the electron is driven into the n-type semiconductor. Because the electrons are the majority carriers, equilibration results in an increase of the electron concentration in the n-type layer over the whole area of the device. Consequently, in a metal salt solution the surface is metallized uniformly, even when only a part of the junction is illuminated; metal deposition is induced by light but is not photoselective.

Here we show that, due to the low mobility of charge carriers in an amorphous semiconductor, photoselective deposition can be achieved with a p-i-n solar cell. Amorphous silicon solar cells consist of a relatively thick intrinsic layer, sandwiched between an $n^{+}$. and $\mathrm{a} \mathrm{p}^{+}$-layer. ${ }^{3}$ In Fig. 1 a schematic energy level diagram of a p-i-n structure in contact with a solution containing metal ions is shown. Most of the light is absorbed in the intrinsic layer; the electric field helps separate the photogenerated electron-hole pair. The electron migrates toward the surface, where it induces the deposition of metal. In contrast to the crystalline silicon case, we expect the electrons to equilibrate slowly; the low mobility of the majority carriers in amorphous silicon together with the limited thickness of the n-type layer limits the lateral mobility of the photogenerated electrons. As a consequence, the reduction of metal ions should

\footnotetext{
* Electrochemical Society Active Member.
}

only occur close to where the carriers are generated, giving metal deposition only in illuminated areas, similar to the p-type semiconductor/electrolyte junction.

State-of-the-art a-Si: $\mathrm{H} \mathrm{p}^{+}-\mathrm{j}-\mathrm{n}^{+}$structures were formed by plasma-enhanced vapor deposition on glass substrates coated with a transparent conducting oxide (TCO). The thickness of the $\mathrm{p}^{+}, \mathrm{i}-$, and $\mathrm{n}^{+}$-layers were 10,500 , and $20 \mathrm{~nm}$, respectively; the effective doping density of the n-type and p-type layer was in the order of $10^{17} \mathrm{~cm}^{-3}$. The TCO was contacted with silver epoxy and both the sample edges and the TCO coated substrate were insulated from the solution using adhesive PTFE (Teflon) tape or Apiezon wax. Gold and nickel layers were deposited from an aqueous $0.01 M$ $\mathrm{KAu}(\mathrm{CN})_{2}, 0.2 \mathrm{M}$ citric acid, $0.2 \mathrm{M}$ sodium citrate solution, ${ }^{1}$ and $0.5 \mathrm{M} \mathrm{NiSO}_{4} \cdot 6 \mathrm{H}_{2} \mathrm{O}$ solution in ethylene glycol, ${ }^{4}$ respectively. Electrochemical experiments were performed using an EG\&G Princeton Applied Research (PAR) 366A bipotentiostat in a conventional electrochemical cell containing a Pt counterelectrode and a saturated calomel electrode (SCE) as reference. For illumination a white light source (Schott KL1500) was used. The thickness of the deposited layers was measured using a Tencor Alpha-Step 500 surface profiler. All chemicals were of p.a. grade (Merck).

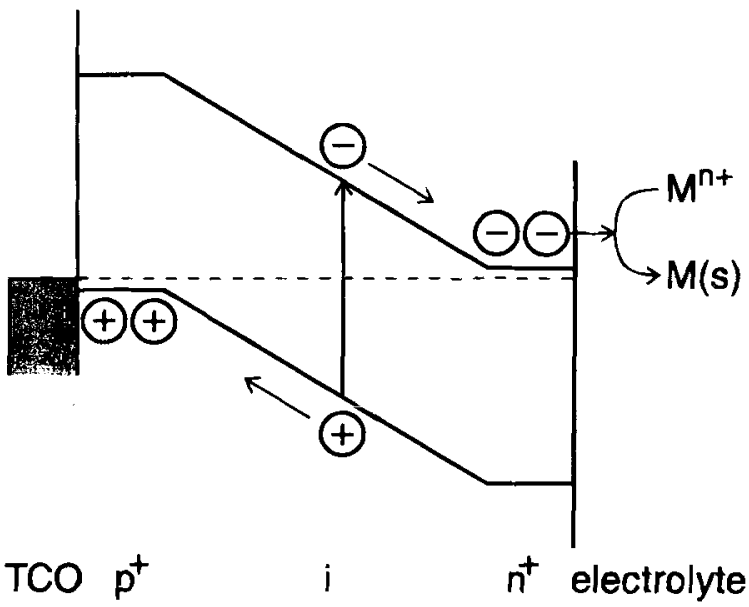

Fig. 1. Schematic energy level diagram of a p-i-n structure illustrating the mechanism of photoinduced metal deposition. 


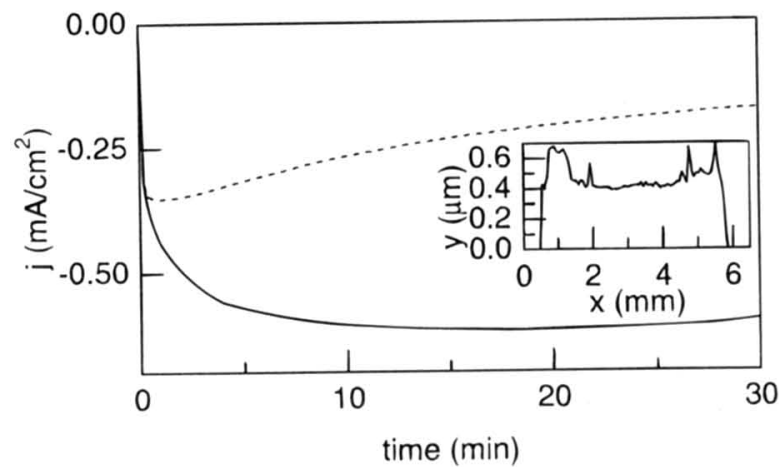

Fig. 2. Photocathodic current as a function of time during the deposition of nickel in a solution of $0.5 \mathrm{M} \mathrm{NiSO}_{4} \cdot 6 \mathrm{H}_{2} \mathrm{O}$ in ethylene glycol; the applied potential was $-0.6 \mathrm{~V}$ vs. SCE. The sample was illuminated either from the substrate side (solid line) or from the solution side (dashed line) for $\mathbf{3 0} \mathrm{min}$. The inset shows a surface profile of the sample illuminated from the substrate side.

To prevent extensive hydrogen evolution as a result of reduction of water, the applied potential was limited to $-0.75 \mathrm{~V} v s$. SCE for gold and $-0.6 \mathrm{~V} v s$. SCE for nickel. In Fig. 2 the photocathodic current during nickel deposition is given as a function of time. When the sample is illuminated through the glass substrate, the current increases and becomes approximately constant after $5 \mathrm{~min}$. The total charge passed through the electrode in $30 \mathrm{~min}$ amounts to $1.04 \mathrm{C} / \mathrm{cm}^{2}$, which corresponds to a $356 \mathrm{~nm}$ thick nickel layer assuming a literature density of $8.9 \mathrm{~g} / \mathrm{cm}^{3}$. The measured thickness, as shown in the inset in Fig. 2, is approximately $20 \%$ larger. Presumably, this is because the electrochemically grown layer is not uniform and exhibits some porosity. When the electrode is illuminated from the solution side, the current first increases but quickly reaches a maximum and then decreases slowly with time. As the thickness of the metal layer increases, the light is reflected or absorbed by the metal layer, giving rise to a lower photocathodic current. Nickel layers grown in this way seem to be less uniform than the layers formed by illumination from the TCO side.

The photoselectivity of the metal deposition is clearly illustrated in Fig. 3, which shows photographic images of gold patterns grown on the amorphous silicon $\mathrm{p}$-i-n electrodes by selective illumination with white light. Patterns were obtained by focusing the light to a single spot on the surface (left) or by placing a grid between the light source and the electrode (right). This photoselective metallization occurs only with amorphous silicon $\mathrm{p}$-i-n structures, and not with crystalline silicon $p-n$ junctions. As mentioned in the introduction the electrons in crystalline material, being the majority carriers in the n-type layer, equilibrate immediately and give rise to an increase of the electron concentration over the whole area.

The electrochemically formed gold contacts exhibit poor electrical characteristics; owing to a significant series resistance, the current through gold contacted devices is small. However, nickel layers provide good ohmic contacts. After annealing the device for $1 \mathrm{~h}$ at $250^{\circ} \mathrm{C}$ under ambient conditions, the current-potential characteristics shown in Fig. 4 are obtained. The reverse current is low in the dark and is linearly dependent on the photon flux under illumination. Under forward bias the current increases with potential, as expected. Prior to annealing the samples the characteristics under reverse bias resemble those of Fig. 4, while the forward current is very small. Apparently, the $n$-type amorphous silicon/nickel interface forms a rectifying junction. This indicates that annealing the deposited nickel contacts is essential. Furthermore, the adhesion of the metal layers to the amorphous silicon surface is considerably improved by annealing. Most probably, a nickel silicide is formed.

In conclusion, we have described a novel and simple method for depositing metal patterns on amorphous silicon p-i-n solar cell structures by selective illumination in a solution containing metal ions. We have shown here that the technique is applicable to gold and nickel, but it is likely that other metals like silver or zinc can be deposited in a similar way.

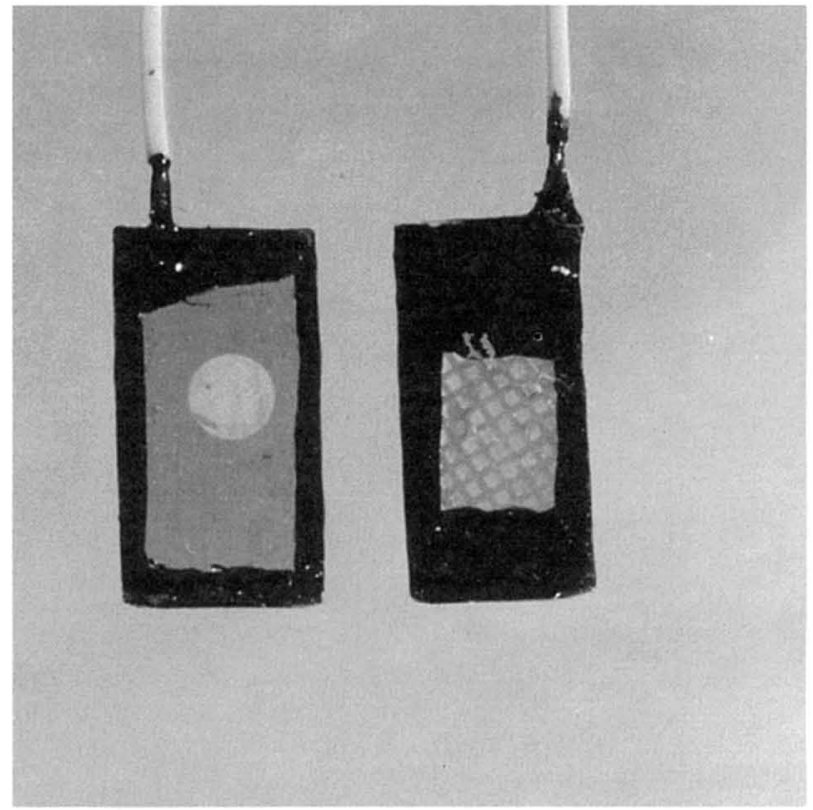

Fig. 3. Image of gold patterns photoselectively deposited on amorphous silicon $\mathrm{p}$-i-n structures. To obtain the sample on the left, the light was focused on a single spot, while for the sample on the right a grid was placed between the illumination source and the electrode. The deposition time was 10 $\mathrm{min}$ and the actual size of the samples was $1.2 \times 2.4 \mathrm{~cm}$.

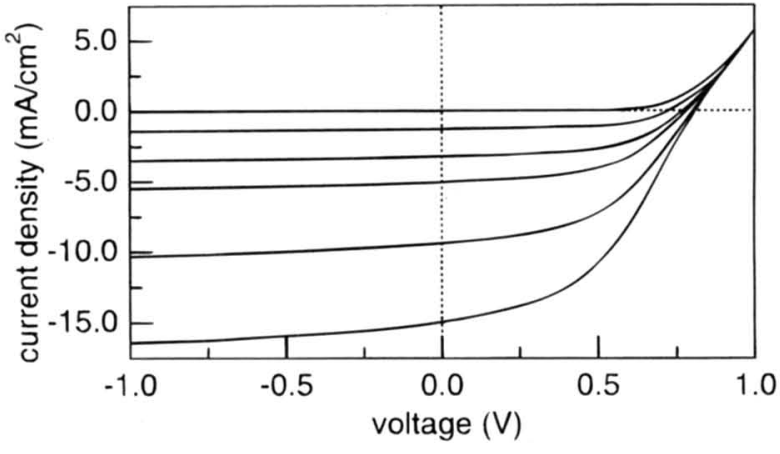

Fig. 4. Current-potential curve of an amorphous silicon p-i-n structure metallized with nickel, after annealing for $1 \mathrm{~h}$ at $250^{\circ} \mathrm{C}$. Measurements were performed using values of the photon flux between $1.2 \times 10^{16}$ and $1.3 \times 10^{17} \mathrm{~cm}^{-2} \mathrm{~s}^{-1}$.

\section{Acknowledgment}

The work described here was supported by the Netherlands Foundation for Chemical Research (SON) with financial aid from the Netherlands Organization for Scientific Research (NWO).

\section{Manuscript received April 7, 1997.}

Utrecht University assisted in meeting the publication costs of this article.

\section{REFERENCES}

1. J. J. Kelly, J. M. G. Rikken, J. W. M. Jacobs, and A. Valster, J. Vac. Sci. Technol. B, 6, 48 (1988).

2. S. R. Morrison, Electrochemistry at Semiconductor and Oxidized Metal Electrodes, Plenum, New York (1980).

3. D. E. Carlson and C. R. Wronski, Appl. Phys. Lett., 28, 671 (1976).

4. E. C. Wurst and E. H. Borneman, J. Appl. Phys., 28, 235 (1957). 